Baltic Astronomy, vol. , , 2014

\title{
THE HALF-CENTURY HISTORY OF STUDIES OF ROMANO'S STAR
}

\author{
Olga Maryeva \\ Special Astrophysical Observatory of the Russian Academy of Sciences, Nizhnii \\ Arkhyz, 369167, Russia; olga.maryeva@gmail.com
}

\begin{abstract}
We present a short review of the observations and simulations of one of the most interesting massive stars, V532, or Romano's star, located in the M33 galaxy.
\end{abstract}

Key words: stars: individual: Romano's star (M33) galaxies: individual: M33 stars: supergiants stars: Wolf-Rayet

\section{INTRODUCTION}

Luminous blue variables (LBVs) are rare objects of very high luminosity ( $\left.10^{6} L_{\odot}\right)$ and mass loss rates $\left(10^{-5} M \div 10^{-4} M_{\odot} \mathrm{yr}^{-1}\right)$, low wind velocities, exhibiting strong irregular photometric and spectral variability Conti, 1984; Humphrevs \& Davidson, 1994; Humphrevs et al., 2014). They are generally believed to be a relatively short evolutionary stage in the life of a massive star, marking the transition from the Main Sequence toward Wolf-Rayet (WR) stars. However, recent studies indicate that progenitors of several supernovae underwent LBV-like eruptions. These studies support the view that at least some LBV stars are the end point of the evolution but not a transition phase. LBVs are rare objects, observations of whose in the Galaxy are inevitably connected with difficulties in determination of the distance and interstellar extinction. Hence, studying these rare objects in nearby galaxies is potentially more prospective. Therefore, investigation of the extragalactic star V5321, which is now classified as LBV/post-LBV star and shows late-WN spectrum, is very important for our understanding of evolution of massive stars in general.

\section{PHOTOMETRIC OBSERVATIONS}

Photometric observations of V532 had been started by Giuliano Romano in Asiago observatory (Italy) in the early 1960s. Romano (1978) demonstrated a light curve from which one may see that stellar magnitude of V532 star irregularly varied between $16^{\mathrm{m}} \cdot 7$ and $18^{\mathrm{m}} \cdot 1$. G. Romano classified V532 as a variable of the Hubble-Sandage type by the shape of the light curve and its colour index (Romano, 1978).

Photometric investigations were continued by Kurtev et al. (2001). Kurtev et al.

\footnotetext{
${ }^{1}$ Romano's star (V532 or GR290) with $\alpha=01: 35: 09.712, \delta=+30: 41: 56.55$ according to SIMBAD data base.
} 
(2001) combined data by G. Romano with their series of photometric observations, carried out during 8 years. They found that during the whole period of study Romano's star displayed two maxima of brightness. The first was near 1970 year and the second - in early 1990s. Moreover Kurtev et al. (2001) discovered shorttimescale variability with amplitude $\sim 0^{\mathrm{m}} .5$ which is typical for an LBV star.

Zharova et al. (2011) investigated photometric variability of V532 using the Moscow collection of photographic plates. They combined these data with new data obtained at Russian 60-cm Zeiss telescope of Sternberg Astronomical Institute and Russian 1-m Zeiss telescope of Special Astrophysical Observatory (SAO) and with photometric data published earlier by Romano (1978); Humphrevs (1980); Viotti et al. (2006). Thus Zharova et al. (2011) constructed the most comprehensive light curve which covers 50 years of observations. The light curve shows that V532 exhibits irregular light variations with different amplitudes and time scales. Generally, the star shows large and complex wave-like variations, with duration of the waves amounting to several years. Four maxima of the waves were observed (Zharova et al., 2011). In general, its variability is irregular, with the power spectrum fairly approximated by a red power-law spectrum (Abolmasov, 2011).

In late 2010 small brightening of Romano's star was detected, the star again reached $17^{\mathrm{m}} .8$ in $V$-band (Calabresi et al., 2014). Calabresi et al. (2014) reported that after a moderate luminosity maximum at the beginning of 2011, the Romano's star has reached a new deep minimum with $\mathrm{V}=18^{\mathrm{m}} .7$ and $\mathrm{R}=18^{\mathrm{m}} \cdot 4$ in December 2013 , which appears to be the deepest so far recorded in its known light curve history.

Moreover photometric observations of V532 were carried out in the infrared range. Stellar magnitudes are $\mathrm{J}=16^{\mathrm{m}} .8, \mathrm{H}=16^{\mathrm{m}} .87$ and $\mathrm{K}=16^{\mathrm{m}} .8$ according to 2MASS survey (Cutri et al., 2003), $16^{\mathrm{m}} .3$ and $15^{\mathrm{m}} .9$ in $3.6 \mu \mathrm{m}$ and $4.5 \mu \mathrm{m}$ bands, respectively, according to the data of Spitzer space telescope (McQuinn et al., 2007).

\section{SPECTRAL OBSERVATIONS}

Spectral observation of Romano's star began only in 1992. T. Szeifert obtained the first optical spectrum of Romano's star at the 3.5-m Calar Alto telescope (Szeifert, 1996). Red range of the spectrum is shown in Szeifert (1996) (Figure 5 in the original article). These spectrum was described by T. Szeifert as "Few metal lines are visible, although a late B spectral type is most likely".

Next spectrum was obtained by Sholukhova et al. (1997) on the Russian 6-m telescope. Sholukhova et al. (1997) classified V532 as a WN star candidate based on the similarity of its spectrum to the one of MCA 1B. MCA $1 \mathrm{~B}$ is also located in M33 galaxy and was classified as Ofp/WN9 (Willis et al., 1992; Smith et al., 1995). Since 1998 year regular observations of V532 are being carried out in SAO (Fabrika et al., 2005; Sholukhova et al., 2011). Sholukhova et al. (2011) published detail series of spectral observations.

Since 2003 spectral observations are being carried by Italian astronomers on telescopes of Cima Ekar (Asiago) and Loiano (Bologna) observatories (Polcaro et al., 2003; Viotti et al., 2006; Polcaro et al., 2011). Moreover spectra of V532 at two important extrema - at the minimum of brightness in 2008 and at the moderate luminosity maximum of 2010 - were described by Polcaro et al. (2011), Maryeva \& Abolmasov (2010) and Clark et al. (2012), respectively. 


\section{CIRCUMSTELLAR NEBULA}

Nebular lines of [OIII] $\lambda \lambda 4959,5007,[\mathrm{NII}] \lambda \lambda 6548-83$, [ArIII] $\lambda 7135$ and [FeIII] $\lambda \lambda 4658,4701$ are clearly seen in the spectrum of V532 (Marveva \& Abolmasov, 2010). Marveva \& Abolmasov (2010) estimated the size and the mass of the V532 nebula and concluded that parameters of the nebula are, by an order of magnitude, consistent with these typical for ejecta of LBV stars. The emitting gas was probably ejected during one or several outburst events at wind velocities of about $100 \mathrm{~km} \mathrm{~s}^{-1}$. The dust circumstellar envelope has not been detected (Humphreys et al., 2014).

\section{STUDIES OF SPECTRAL VARIABILITY AND SPECTRAL CLASSIFICATION}

The first investigations of spectral variability were started by Viotti et al. (2006, 2007). Using five spectra acquired in $2003 \div 2006$ Viotti et al. $(2006,2007)$ found an anti-correlation between equivalent widths of the Wolf-Rayet blue bump at $4630 \div 4686 \AA \AA$ and the visual luminosity. Maryeva \& Abolmasov (2010) classified archived spectra of V532 and demonstrated an evidence for a correlation between spectral type and visible magnitude. Also it was displayed that in the deep minimum (2008 year) the spectrum of Romano's star became similar to one of WN8 star type (Maryeva \& Abolmasov, 2010).

It is easy enough to classify every single spectrum obtained at various moments of time, but there is no consensus among the researchers on the type of the star. As stated above, G. Romano classified the star as a Hubble-Sandage variable. Humphreys \& Davidson (1994) on the basis of its variability classified the star as an LBV candidate. Polcaro et al. (2003) estimated the bolometric absolute magnitude of the object as $M_{b o l} \approx-10^{\mathrm{m}} \cdot 4$, using bolometric correction "of at least -3 mag" and distance modulus $m-M=24$ m. They classify V532 as an LBV because the object fulfills all the criteria of Humphreys \& Davidson (1994).

An additional argument for LBV/post-LBV status of V532 is its location in the galaxy. V532 does not belong to any OB-association of M33 galaxy. Smith \& Tombleson (2014) concluded that LBVs systematically avoid clusters of O-type stars, and they are almost never closely associated with O-type stars of similar (presumed) initial mass.

However, Polcaro et al. (2011) concluded that bolometric luminosity has significantly changed during minimum phase of 2008 year and suggested that Romano's star now is about to end its LBV phase and to become a late WN type star.

In a recent article Humphreys et al. (2014) picked out one more feature of LBV star - its slow stellar wind. LBVs have low wind speeds in their hot, quiescent or visual minimum state, compared to the B-type supergiants and Of/WN stars which they spectroscopically resemble (Humphreys et al., 2014). Humphreys et al. (2014) concluded that in spite of Romano's star having slow wind it is not an LBV star on all the characteristics. Most probably Romano's star has already passed its LBV phase, and is a post-LBV star now.

\section{NUMERICAL SIMULATION}

Today numerical modeling provides most complete information about parameters of stellar atmospheres. The first modeling of V532 atmosphere was performed by Marveva \& Abolmasov (2012a). Non-LTE radiative transfer code CMFGEN (Hillier \& Miller, 1998) was used for the analysis. To accurately determine 
Table 1: Derived properties of V532. H/He denotes hydrogen number fraction relative to helium, $f$ is a filling factor.

\begin{tabular}{lcccccccc}
\hline Star & $\begin{array}{c}\text { Sp. } \\
\text { type }\end{array}$ & $\begin{array}{c}T_{\text {eff }} \\
{[\mathrm{kK}]}\end{array}$ & $\begin{array}{c}R_{2 / 3} \\
{\left[\mathrm{R}_{\odot}\right]}\end{array}$ & $\begin{array}{c}\log L_{*} \\
{\left[\mathrm{~L}_{\odot}\right]}\end{array}$ & $\begin{array}{c}\dot{M}_{c l}, 10^{-5} \\
{\left[\mathrm{M}_{\odot} \mathrm{yr}^{-1}\right]}\end{array}$ & $\mathrm{f}$ & $\begin{array}{c}v_{\infty} \\
{[\mathrm{km} / \mathrm{s}]}\end{array}$ & $\mathrm{H} / \mathrm{He}$ \\
\hline $2005^{1}$ & WN11h & 20.4 & 69.1 & 5.89 & 4.5 & 0.5 & 200 & 1.4 \\
$2008^{1}$ & WN8h & 31.7 & 23.9 & 5.72 & 2 & 0.1 & 360 & 1.9 \\
$2010^{2}$ & WN10h & 26 & 41.5 & 5.85 & 2.18 & 0.25 & 265 & 1.5 \\
2014 & WN8h & 32.7 & 22 & 5.72 & 1.7 & 0.1 & 400 & 1.9 \\
\hline 1
\end{tabular}

of the luminosity the model flux was recalculated for the distance of M33 for every model. Distance to M33 was adopted as $D=847 \pm 60 \mathrm{kpc}$, which gives a distance modulus of $(m-M)=24.64 \pm 0.15^{\mathrm{m}}$ (Galleti et al., 2004). Then, the simulated spectra were convolved with B- and V-band sensitivity curves. The resulting fluxes were converted to magnitudes (Lang, 1974) and compared to the photometrical data.

Maryeva \& Abolmasov (2012a) investigated the optical spectra of Romano's star in two different states, the brightness minimum of 2008 ( $B=18.5 \pm 0.05 \mathrm{mag})$ and a moderate brightening in $2005(B=17.1 \pm 0.03 \mathrm{mag})$. Figure 1 shows the observed spectra of V532 at different phases and the best-fit model spectra. Stellar parameters derived for both models are given in Table 1.

Main result of the work of Maryeva \& Abolmasov (2012a) is that the bolometric luminosities of V532 were different in 2005 and 2008. The bolometric luminosity of V532 in $2005\left(L_{*}=7.5 \cdot 10^{5} L_{\odot}\right)$ is 1.5 times higher. This result confirms the conclusion of Polcaro et al. (2011).

Clark et al. (2012) modelled the spectrum of Romano's star obtained in September 2010 when the V-band magnitude of the object was between $17^{\mathrm{m}} \cdot 75$ and $17^{\mathrm{m}} .85$. They also used CMFGEN code (Hillier \& Miller, 1998). For comparison the results of Clark et al. (2012) are also listed in Table 1. Clark et al. (2012) inferred (see Table 1$)$ that the physical properties $\left(T_{e f f}, v_{\infty}\right.$, filling factor $f$ ) of Romano's star in 2010 are a smooth progression between photometric minima and maxima. We assume that values of $\mathrm{H} / \mathrm{He}$ agree within the limits of errors. It may be seen from the table that values of luminosities in 2008 and 2010 are different. However, it must be noted that Clark et al. (2012) assumed that the distance to M33 is equal to $964 \mathrm{kpc}$, while Maryeva \& Abolmasov (2012a) took the distance of $847 \mathrm{kpc}$. If we recalculate the luminosity found by Clark et al. $L_{*}=7 \cdot 10^{5} \mathrm{~L}_{\odot}$ to distance $847 \mathrm{kpc}$ we get that luminosity of V532 is $L_{*}=5.4 \cdot 10^{5} \mathrm{~L}_{\odot}$ and it did not change after 2008 within the error limits $\left(L_{*}=5.2 \pm 0.2 \cdot 10^{5} \mathrm{~L}_{\odot}\right.$, Marveva \& Abolmasov $(2012 \mathrm{a}))$.

\section{NEW OBSERVATIONS}

In August 2014 we have obtained a new spectrum of V532 on the Russian 6-m telescope with the SCORPIO multi-mode focal reducer in the long-slit mode Afanasiev \& Moiseev, 2005). The spectrum was reduced using the ScoRe package written in IDL language. The package includes all the standard stages of long-slit data reduction process. 

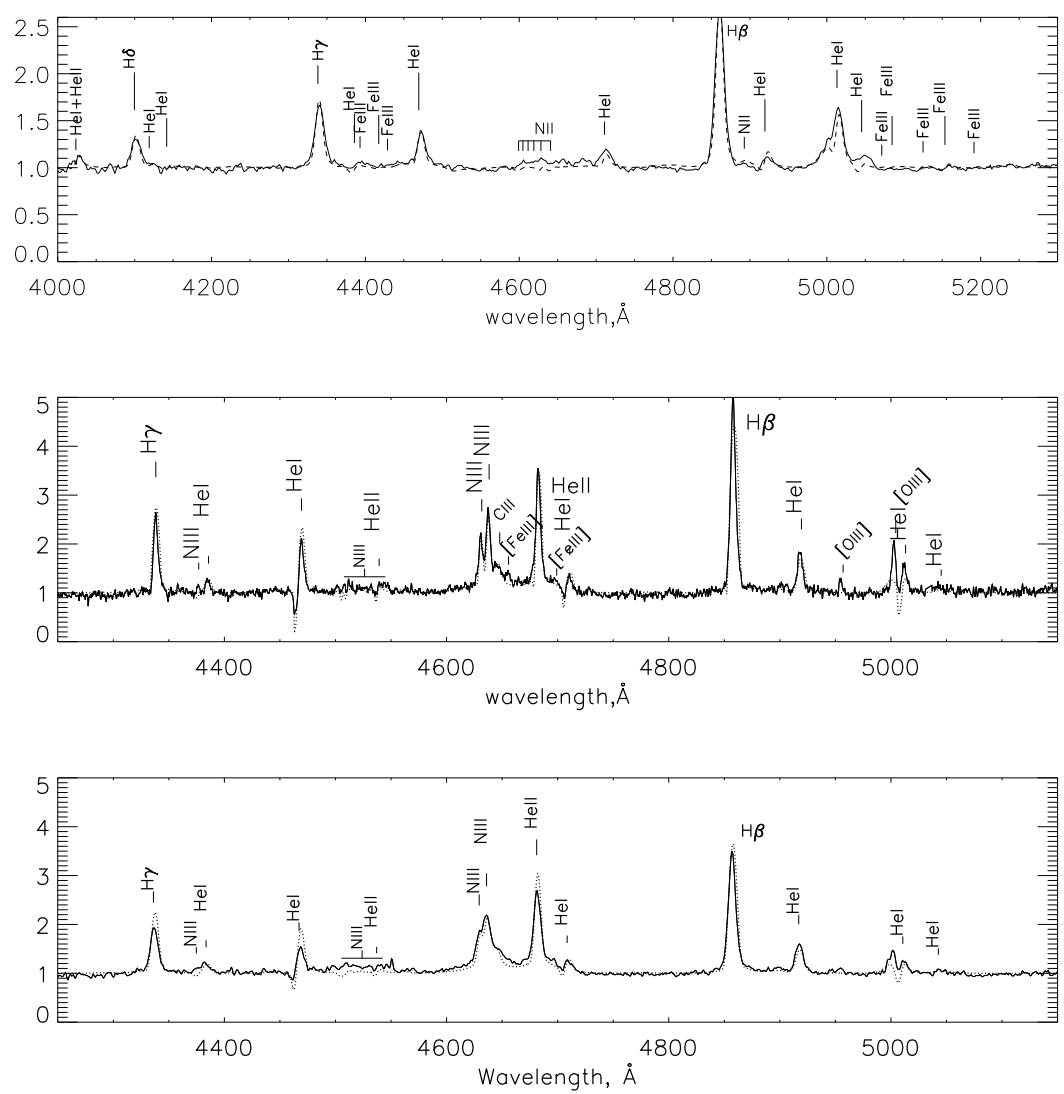

Fig. 1. The normalized optical spectra (solid line) compared with the best-fit CMFGEN models (dashed line). Top panel: the spectrum obtained in February 2006 when V532 was $17^{\mathrm{m}} .27$ in $\mathrm{V}$ band. Middle panel: the spectrum obtained in October 2007 $\left(V=18^{\mathrm{m}} .68\right)$. Bottom panel: the spectrum obtained in August $2014\left(V=18^{\mathrm{m}} \cdot 7\right)$.

Now V532 is in the minimum of brightness, its visible magnitude is $\mathrm{V}=18^{\mathrm{m}} \cdot 72$ Bottom panel of Figure 1 shows the spectrum obtained in August 2014 and its best-fit model. As one may see from the figure, the new August spectrum is very similar to spectrum obtained in early 2008 . This is verification of hypothesis that the change of the stellar magnitude occurs simultaneously with spectral class changes. The dependence of stellar magnitude on spectral class doesn't vary with time. Therefore we can use this property for studies of the periods for which we have only photometric data.

\section{CONCLUDING REMARKS}

Figure 2 presents the positions of V532 in different phases in the Hertzsprung-

2 Photometric data were kindly provided by Roberto Haver and Massimo Calabresi, scientists of Associazione Romana Astrofili (ARA) 
Russell (HR) diagram according to modeling by Marveva \& Abolmasov (2012a). V532 in maximum of brightness ( $V=17^{\mathrm{m}}$, Feb. 2005) lies on the LBV minimum instability strip. It moves to the "forbidden region" in the minimum of brightness. V532 is the first star which demonstrated the transition from the instability strip to WR region (Maryeva, 2013). The main argument for what V532 is now not an LBV is that it does not exhibit S Dor-like transitions to the cool, dense wind state. Instead, it oscillates between two hot states on the HR diagram characterized by WN spectroscopic features (Humphreys et al., 2014). However there is a spectrum obtained in 1992 which is classified as a late B spectral type (Szeifert, 1996). Maybe this spectrum is a confirmation that V532 in early 90s was actually in a cool state?

Other interesting question is how did the luminosity of Romano's star change before and after moderate brightening in 2005? How did other parameters of the atmosphere behave? To answer these questions, numerical modeling of archive spectra is needed. As mass loss rate depends on luminosity it is necessary to make calculations for different phases in a consistent way.

It is clearly seen from this review that V532 has been primarily studied in the optical range. While its optical monitring is being performed regularly, in other wavebands it has not been studied well. Estimations of X-ray, ultraviolet and radio luminosities of V532 are necessary for precise construction of its spectral energy distribution (SED). Moreover it is interesting to observe variability in different ranges. We consider that investigation of V532 in wide range of wavelenghts is a top priority task.

ACKNOWLEDGMENTS. I would like to thank Roberto Viotti, Roberto Haver and Massimo Calabresi for providing new photometric data and the Referee V.P. Arkhipova for valuable remarks given in the Referee report. The study was supported by the Russian Foundation for Basic Research (projects no. 12-0700739-a, 14-02-31247, 14-02-00291). I thank the Dynasty Foundation for a grant.

\section{References}

Abolmasov P., 2011, New Astronomy, 16, 421

Afanasiev V. L., Moiseev A. V., 2005, Astromy Let., 31, 194

Calabresi M., Rossi C., Gualandi R., et al., 2014, Astro. Telegram, 5846, 1

Clark J. S., Castro N., Garcia M., et al., 2012, A\&A, 541, A146

Conti P. S., 1984, in Maeder A., Renzini A., eds, IAU Symposium Vol. 105, Observational Tests of the Stellar Evolution Theory. p. 233

Crowther P. A., Smith L. J., Hillier D. J., Schmutz W., 1995, A\&A, 293, 427

Crowther P. A., Pasquali A., De Marco O., et al., 1999, A\&A, 350, 1007

Crowther P. A., Smith L. J., 1997, A\&A, 320, 500

Cutri R. M., et al., 2003, VizieR Online Data Catalog, 2246, 0

Fabrika S., Sholukhova O., Becker T., et al., 2005, A\&A437, 217

Galleti S., Bellazzini M., Ferraro F. R., 2004, A\&A, 423, 925

Groh J. H., Damineli A., Hillier D. J., et al., 2009, ApJL, 705, L25

Groh J. H., Hillier D. J., Damineli A., et al., 2009, ApJ, 698, 1698 


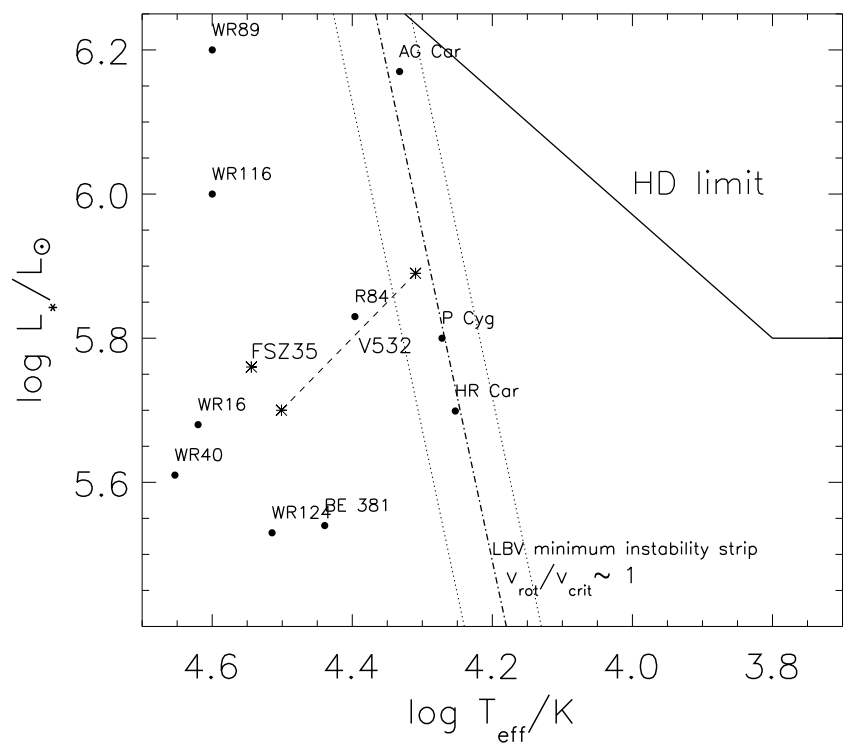

Fig. 2. A schematic of upper part of HR Diagram. Position for the LBV minimum instability strip is provided by dashed-dotted line. The location of the Humphreys-Davidson limit (Humphrevs \& Davidson, 1994) is shown by solid line. Dashed line shows transit V532 across instability strip. WN9 (BE381, R84), WN8 (WR124, WR16, WR40, FSZ35) and LBV (AG Car, HR Car, P Cyg) stars are shown for comparison. Data on these objects were taken from (Crowther \& Smith, 1997), (Crowther \& Smith, 1997), (Crowther et al., 1999), (Herald et al., 2001), (Herald et al., 2001), (Marveva \& Abolmasov, 2012b), (Groh et al., 2009), (Groh et al., 2009), (Naiarro, 2001), consequently.

Herald J. E., Hillier D. J., Schulte-Ladbeck R. E., 2001, ApJ, 548, 932

Hillier D. J., Miller D. L., 1998, ApJ, 496, 407

Humphreys R. M., 1980, ApJ, 241, 587

Humphreys R. M., Davidson K., 1994, Publications of the ASP, 106, 1025

Humphreys R. M., Weis K., Davidson K., et al., 2014, ApJ, 790, 48

Kurtev R., Sholukhova O., Borissova J., Georgiev L., 2001, Rev. Mex., 37, 57

Lang K. R., 1974, Astrophysical formulae: A compendium for the physicist and astrophysicist

Maeder A., Meynet G., 2010, New Astronomy Review54, 32

Maryeva O., 2013, Astronom. and Astrophys. Tr., 28, 35

Maryeva O., Abolmasov P., 2010, Rev. Mex., 46, 279

Maryeva O., Abolmasov P., 2012a, MNRAS, 419, 1455

Maryeva O., Abolmasov P., 2012b, MNRAS, 421, 1189

McQuinn K. B. W., et al., 2007, ApJ664, 850

Najarro F., 2001, in de Groot M., Sterken C., eds, Astronomical Society of the Pacific Conference Series Vol. 233, P Cygni 2000: 400 Years of Progress. p. 133 
Polcaro V. F., Gualandi R., Norci L., et al., 2003, A\&A, 411, 193

Polcaro V. F., Rossi C., Viotti R. F., et al., 2011, AJ, 141, 18

Romano G., 1978, A\&A, 67, 291

Sholukhova O. N., Fabrika S. N., Vlasyuk V. V., Burenkov A. N., 1997, Astromy Let., 23,458

Sholukhova O. N., Fabrika S. N., Zharova A. V., et al., 2011, Astrophys. Bul., 66, 123

Smith N., Conti P. S., 2008, ApJ679, 1467

Smith N., Tombleson R., 2014, ArXiv e-prints

Smith L. J., Crowther P. A., Willis A. J., 1995, A\&A, 302, 830

Szeifert T., 1996, in Vreux J. M., Detal A., Fraipont-Caro D., Gosset E., Rauw G., eds, Liege International Astrophysical Colloquia Vol. 33 of Liege International Astrophysical Colloquia, LBVs and a late WN-star in M 31 and M 33. p. 459

Viotti R. F., Galleti S., Gualandi R., et al., 2007, A\&A, 464, L53

Viotti R. F., Rossi C., Polcaro V. F., et al., 2006, A\&A, 458, 225

Willis A. J., Schild H., Smith L. J., 1992, A\&A, 261, 419

Zharova A., Goranskij V., Sholukhova O. N., Fabrika S. N., 2011, Peremennye Zvezdy Prilozhenie, 11, 11 